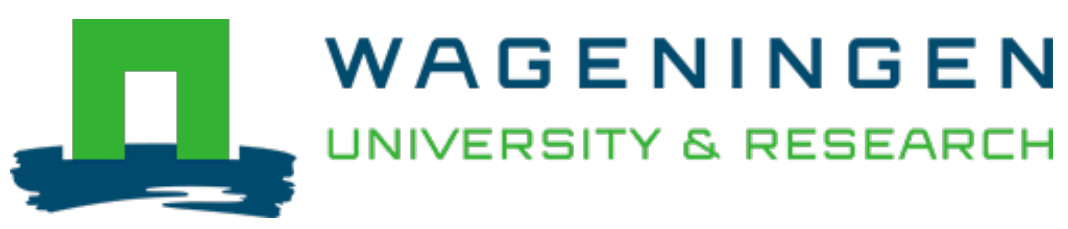

\title{
Opportunities for Products of New Plant Breeding Techniques
}

Trends in Plant Science

Schaart, J.G.; Wiel, C.C.M.; Lotz, L.A.P.; Smulders, Rene

https://doi.org/10.1016/j.tplants.2015.11.006

This article is made publicly available in the institutional repository of Wageningen University and Research, under the terms of article $25 \mathrm{fa}$ of the Dutch Copyright Act, also known as the Amendment Taverne. This has been done with explicit consent by the author.

Article $25 \mathrm{fa}$ states that the author of a short scientific work funded either wholly or partially by Dutch public funds is entitled to make that work publicly available for no consideration following a reasonable period of time after the work was first published, provided that clear reference is made to the source of the first publication of the work.

This publication is distributed under The Association of Universities in the Netherlands (VSNU) 'Article $25 \mathrm{fa}$

implementation' project. In this project research outputs of researchers employed by Dutch Universities that comply with the legal requirements of Article $25 \mathrm{fa}$ of the Dutch Copyright Act are distributed online and free of cost or other barriers in institutional repositories. Research outputs are distributed six months after their first online publication in the original published version and with proper attribution to the source of the original publication.

You are permitted to download and use the publication for personal purposes. All rights remain with the author(s) and / or copyright owner(s) of this work. Any use of the publication or parts of it other than authorised under article $25 \mathrm{fa}$ of the Dutch Copyright act is prohibited. Wageningen University \& Research and the author(s) of this publication shall not be held responsible or liable for any damages resulting from your (re)use of this publication.

For questions regarding the public availability of this article please contact openscience.library@wur.nl 


\section{Trends in Plant Science}

\section{Review \\ Opportunities for Products of New Plant Breeding Techniques}

\author{
Jan G. Schaart, ${ }^{1, \star}$ Clemens C.M. van de Wiel, ${ }^{1}$ \\ Lambertus A.P. Lotz, ${ }^{2}$ and Marinus J.M. Smulders ${ }^{1}$
}

Various new plant breeding techniques (NPBT) have a similar aim, namely to produce improved crop varieties that are difficult to obtain through traditional breeding methods. Here, we review the opportunities for products created using NPBTs. We categorize products of these NPBTs into three product classes with a different degree of genetic modification. For each product class, recent examples are described to illustrate the potential for breeding new crops with improved traits. Finally, we touch upon the future applications of these methods, such as cisgenic potato genotypes in which specific combinations of Phytophthora infestans resistance genes have been stacked for use in durable cultivation, or the creation of new disease resistances by knocking out or removing Sgenes using genome-editing techniques.

\section{New Plant Breeding Techniques Facilitate Breeding of Improved Crop Varieties}

Crop improvement is an important endeavor if we are to meet the demands of a growing population (a worldwide population of 9 billion people is projected for 2050), for which food production needs to be increased, while at the same time the environmental impact of food production needs to be reduced. To respond adequately, we should optimally apply all existing tools to breed improved crops and maximize any potential future applications for increasingly sustainable food production.

Plant breeding has resulted in numerous improved food, feed, ornamental, and industrial crop varieties and traditional breeding based on crossing and selection remains an important activity for crop improvement. Although the efficiency of crossing and selection has been improved by using marker-assisted selection, it faces limitations in crops with complex genetics (e.g., due to polyploidy, heterozygosity, or self-incompatibility) or a long generation time (e.g., fruit trees). In addition, the search for useful genetic variation is often laborious, and introgressing such variation from wild relatives into the cultivated germplasm through crossing can be tedious.

Mutation breeding using chemical mutagens or ionizing radiation is used to create new genetic variation. The selection of mutants was originally based on phenotypic variation, but the availability of new advanced genomics technologies has facilitated the selection of plants with desired mutations. Nevertheless, screening for mutants remains time consuming and expensive, and requires large populations. Once mutant plants have been selection, subsequent breeding steps are required to achieve homozygous mutations and to remove undesired mutations. Although mutation breeding is regarded as genetic modification (GM), it is exempted from GM legislation by annex IB of Directive 2001/18/EC.
Trends

Several NPBTs are currently being implemented and represent a significant step forward for crop improvement compared with traditional breeding

NPBTs make use of a genetic modification step, but the resulting endproducts do not contain any foreign genes. Consequently, NPBT products are genetically similar to, or may be even indistinguishable from, traditionally bred plants.

Recent studies show the remarkable potential of NPBTs for the production of innovative crop varieties.

${ }^{1}$ Wageningen UR Plant Breeding, Droevendaalsesteeg 1, NL-6708 PB Wageningen, The Netherlands ${ }^{2}$ Wageningen UR Agrosystems Research, Droevendaalsesteeg 1 , NL-6708 PB Wageningen, The Netherlands

${ }^{*}$ Correspondence: jan.schaart@wur.nl (J.G. Schaart). 


\section{Trends in Plant Science}

Over the past 15 years, several new techniques have been developed and are being implemented to facilitate breeding of improved crop varieties. Compared with traditional breeding, these techniques increase the precision of making changes in the genomes and thereby reduce the time and effort that is needed to produce varieties that meet new requirements. A common denominator of these techniques is that they make use of a GM step, but result in products in which no foreign genes (i.e., genes other than from the species itself or from cross-compatible species) are present. The exception is the use of genome editing to insert transgenes using sequence-specific nuclease technology (SSN-3; see Glossary), where the innovation is that genes can be inserted at a precise, predefined location, without the need for T-DNA border sequences, occurrence of small deletions, and so on.

In 2007, a New Techniques Working Group was established by the European Commission with the task of evaluating these new techniques of GM with respect to the current European Union (EU) genetically modified organism (GMO) legislation. This working group identified several NPBTs [1], and a short definition of each is given in the Glossary. In addition to these NPBTs, we also consider a new development, which we call 'induced early flowering', as a NPBT.

The regulatory issues with regard to biosafety of NPBT products are complex, because the techniques vary greatly with regards to the technologies used and the impact of the applied modification on the plant genome. Some of the end products are indistinguishable from conventional plant breeding products. In the EU, the current GM legislation (European Directive 2001/18/EC) is process based, triggered by the use of a GM step. A product-based approach would be more flexible when new technological developments are applied. Such an approach is also more consistent with current scientific understandings of the risks involved with GM and would provide a more flexible approach to regulation. The regulatory issues were recently reviewed by Araki et al. [2], who addressed the fact that some of these techniques blur the current boundaries of product- and process-based regulations, but how they do that also differs between jurisdictions. The lack of clarity regarding the regulatory issues undermines confidence in the new technologies and, therefore, stifles investment and innovation [3]. Other recent reviews have also focused on regulatory uncertainty [3], social acceptance [4], and the technical side of NPBTs, in particular on current developments in genome-editing technology (e.g., [5-7]).

Here, we focus on the potential for innovative crop varieties made by using these new techniques, as has already been demonstrated in recent studies. We survey the type of products that can be made with various NPBTs and provide three product classes with a different degree of modification. For each product class, relevant examples of plants recently produced by NPBTs are described to illustrate the potential for new crops with improved traits. Finally, we touch upon possible future applications for these methods.

\section{NPBTs Produce Three Types of Improved Plant}

Products from NPBTs may be grouped into three classes: (i) improved plants that contain a new DNA fragment (usually a new gene); (ii) improved plants that do not contain a new DNA fragment, but have a mutation or modification in their own DNA; and (iii) improved plants that do not contain a new DNA fragment or any modification of their DNA (Figure 1, Key Figure). Below, we describe these different product classes.

\section{NPBT Products that Contain New DNA Fragments}

Products made with cisgenesis, intragenesis and specific cases of genome editing using SSN3 technology contain new DNA fragments (Table 1, Improved plant 1). Both cisgenesis and intragenesis are concepts relevant to genetic transformation technology and concern the origin of the inserted DNA. For cisgenesis, a copy of a complete natural gene, including the promoter
Glossary

Agroinfiltration: a technique using Agrobacterium as a tool to achieve temporary and local expression of genes in plant tissue. Agroinfiltration is applied for testing the reaction of target plants to transgenic proteins, or for functional gene analysis in plants.

Cisgenesis: the production of plants by genetic modification using only genes from the species itself or from a species that can be crossed with this species using traditional methods (for overview of these traditional methods, see iv). The genes used are added as an extra copy and are natural variants.

Grafting on GM rootstock: the top of a nonGM plant is grafted on a GM rootstock. In such a graft, the scion may benefit from traits conferred by transgenes in the rootstock, but its products do not contain the transgene itself.

Induced early flowering: a recombinant gene that induces flowering is introduced into a plant. The gene confers flowering of the seedlings in the first year and, thus, speeds up breeding in species with a long generation time. The recombinant gene is removed in the final cross(es) leading to the end product that will be marketed. In an alternative approach, the gene is delivered in the form of a virus [44]. Intragenesis: similar to cisgenesis, because it uses only gene sequences from the species itself or from a crossable species, but the genes introduced are novel combinations of functional elements originating from different genes.

Oligo-directed mutagenesis

(ODM): introduces specific mutations at defined locations in the plant genome. Synthetic oligonucleotides homologous to the target DNA, but containing mismatches, are introduced into plant cells. The mismatches in base pairing between the oligonucleotide and target DNA are corrected by the native repair mechanism of the plant, resulting in point mutations in the targeted DNA. Reverse breeding: an approach for the generation of homozygous parental lines from any heterozygous line. By crossing these homozygous parental lines, the heterozygous line is reproduced as a form of hybrid seed production. Reverse breeding may make use of genetic 


\section{Trends in Plant Science}

\section{Key Figure}

\section{Outline of Process Followed by New Plant Breeding Techniques}

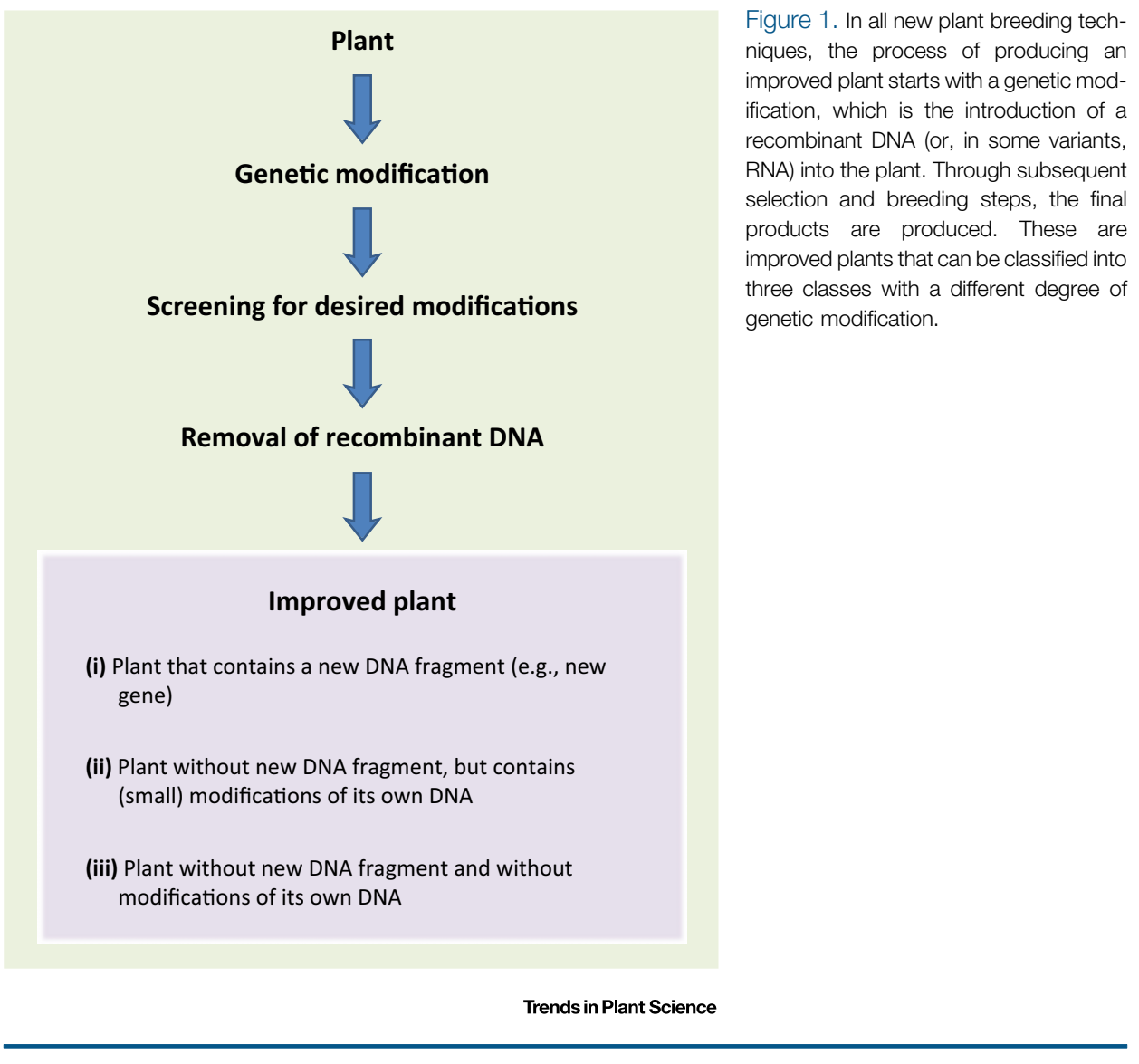

and terminator sequences, from the sexual compatible gene pool is introduced. This is often an allele with beneficial characteristics. Intragenesis uses a novel combination of gene elements that all originate from the species or from a cross-compatible species. An example of a novel combination is a gene whose coding sequence is combined with new regulatory gene elements (i.e., promoter and terminator sequences) of another gene to change its pattern of expression. SSN-3 technology enables the insertion of new DNA fragments at a predetermined location in the genome. The class of NPBT products in this section includes plants that are produced using SSN-3 technology in which the targeted integration of a cis-, intra-, or transgene is into a socalled 'safe harbor' locus. This is a chromosomal location where genes can integrate and function in a predictable manner. AAVS1 and Rosa26 are well-validated safe harbor loci in the human and mouse genome, respectively, and are generally used in therapeutic research, because they permit stable gene expression of inserted transgenes [8]. In plants, no such general safe harbor sites have been identified. The only exception is in rice, where a noncoding region with high expression of the integrated transgenes was considered as a safe harbor locus for gene insertion [9]. SSN-3 for targeted integration of a transgene has been described for insertional inactivation of an endogenous gene [10]. It has also been used for sequential transgene stacking at a specific chromosomal locus [11]. The advantage of gene stacking is modification for the suppression of meiotic recombination enabling complementary homozygous lines to be obtained. The recombinant gene is removed in the crossing. RNA-directed DNA methylation (RdDM): induces transcriptional gene silencing via methylation of the target gene promoter sequence by RNA interference (RNAi). Transgenes encoding RNAs that are homologous to promoter regions are delivered into the plant cell. The RNA-induced silencing complex (RISC) of the plant is triggered by the RNAs and induces methylation of the homologous target promoter sequence. Ultimately, this results in silencing of the target gene expression.

Sequence-specific nuclease (SSN) technology: using SSN technology, any gene of interest can be stably knocked out, mutated, or replaced. SSNs bind to a predefined target DNA sequence at which they induce a double-stranded break (DSB). The type of native repair pathway used for the DSB repair determines the final result, which can be a small deletion or insertion or a precise insertion. Zinc finger nucleases (ZFNs), meganucleases, TALENs and CRISPR-Cas9 are examples of SSNs. SSNs are also called site-directed nucleases (SDNs). The application of SSN technology is also known as 'genome editing'.

TThe description of these NPBTs is based on the definitions and terms described elsewhere [1] iii. 


\section{Trends in Plant Science}

Table 1. Classification of Final Products From NPBTs $s^{a, b}$

\begin{tabular}{|c|c|c|c|c|c|}
\hline \multirow[t]{3}{*}{ Technique } & \multicolumn{3}{|c|}{ What is the Final Product after Breeding? } & \multirow{3}{*}{$\begin{array}{l}\text { NPBT Products with } \\
\text { Improved Traits }\end{array}$} & \multirow[t]{3}{*}{ Refs } \\
\hline & $\begin{array}{l}\text { Improved } \\
\text { Plant } 1\end{array}$ & $\begin{array}{l}\text { Improved } \\
\text { Plant } 2\end{array}$ & $\begin{array}{l}\text { Improved } \\
\text { Plant } 3\end{array}$ & & \\
\hline & $\begin{array}{l}\text { Plant with } \\
\text { New Genes } \\
\text { at a New } \\
\text { Chromosomal } \\
\text { Locus }\end{array}$ & $\begin{array}{l}\text { Plant without } \\
\text { New Genes } \\
\text { but with a } \\
\text { Mutation }\end{array}$ & $\begin{array}{l}\text { Plant without } \\
\text { New Genes or } \\
\text { Modifications }\end{array}$ & & \\
\hline \multirow[t]{3}{*}{ Cisgenesis } & \multirow[t]{3}{*}{ Yes $^{\mathrm{C}}$} & \multirow[t]{3}{*}{ Not } & \multirow[t]{3}{*}{ Not } & $\begin{array}{l}\text { Late blight resistance in } \\
\text { potato }\end{array}$ & [14] \\
\hline & & & & Scab resistance in apple & [15] \\
\hline & & & & High phytase activity in barley & [17] \\
\hline Intragenesis & Yes $^{\mathrm{C}}$ & Not & Not & $\begin{array}{l}\text { Potatoes with reduced } \\
\text { acrylamide levels after } \\
\text { processing }\end{array}$ & [13] \\
\hline \multirow[t]{4}{*}{$\begin{array}{l}\text { SSN technology } \\
\text { (SSN-1 and SSN-2) }\end{array}$} & \multirow[t]{4}{*}{ Not } & \multirow[t]{4}{*}{ Yes } & \multirow[t]{4}{*}{ Not } & $\begin{array}{l}\text { Bacterial leaf blight in rice } \\
\text { (SSN-1) }\end{array}$ & [18] \\
\hline & & & & $\begin{array}{l}\text { Powdery mildew resistance } \\
\text { in bread wheat (SSN-1) }\end{array}$ & [19] \\
\hline & & & & $\begin{array}{l}\text { Soybean with chanced fatty } \\
\text { acid profile (SSN-1) }\end{array}$ & [20] \\
\hline & & & & $\begin{array}{l}\text { Potatoes with reduced } \\
\text { acrylamide levels after } \\
\text { processing (SSN-1) }\end{array}$ & [21] \\
\hline $\begin{array}{l}\text { SSN technology } \\
\text { (SSN-3) }\end{array}$ & Yes $^{d}$ & Yes $^{e}$ & Yes $^{f}$ & $\begin{array}{l}\text { Maize with reduced phytate } \\
\text { content and herbicide } \\
\text { tolerance }\end{array}$ & [10] \\
\hline $\begin{array}{l}\text { Oligo-directed } \\
\text { mutagenesis (ODM) }\end{array}$ & Not & Yes & Not & Imidazolinone-tolerant maize & [25] \\
\hline $\begin{array}{l}\text { RNA-dependent } \\
\text { DNA methylation }\end{array}$ & Not & Not & Yes & NR & \\
\hline Reverse breeding & Not & Not & Yes & NR & \\
\hline $\begin{array}{l}\text { Induced early } \\
\text { flowering }\end{array}$ & Not & Not & Yes & $\begin{array}{l}\text { Apple lines with stacked scab } \\
\text { and fire blight resistances }\end{array}$ & [26] \\
\hline $\begin{array}{l}\text { Grafting on GM } \\
\text { rootstock }\end{array}$ & Not & Not & Yes & $\begin{array}{l}\text { Prunus necrotic ringspot } \\
\text { virus resistance in sweet- } \\
\text { cherry scions }\end{array}$ & [30] \\
\hline
\end{tabular}

aYes, improved plants produced; Not, this type of improved plant is not produced using this technique.; NR, none reported. ${ }^{\mathrm{b}}$ Modified after [1].

'New DNA originates from the same or closely related species.

'Targeted integration of cisgenes, intragenes or transgenes at a 'safe harbour' location.

${ }^{\mathrm{e}}$ Gene replacement with a modified (artificially changed) allele (modified cisgene).

${ }^{\mathrm{f} G e n e ~ r e p l a c e m e n t ~ w i t h ~ a ~ n a t u r a l ~ a l l e l e ~(c i s g e n e) . ~}$

that, during subsequent crossings, the stacked transgenes segregate as a single locus. SSN-3mediated integration of cis- or intragenes using Agrobacterium for transformation can occur without the integration of the T-DNA border and vector backbone sequences and the small deletions that typically surround T-DNA insertions, all of which are undesirable in the resulting modified plants. However, the use of SSN-3 technology for introducing cis- or intragenes has not yet been reported. The SSN-3-mediated targeted integration examples described so far rely on the use of selectable transgenes, which is a consequence of the low efficiency of gene targeting via homologous recombination. For targeted integration of random cis- or intragenes, an improvement of the efficiency of the integration method is required. 


\section{Trends in Plant Science}

\section{Application of Cisgenesis and Intragenesis}

Several examples following the cisgenesis or intragenesis concept have been described and reviewed in [12]. The examples described below all used Agrobacterium-mediated transformation, with or without the application of a selection gene. Various approaches used to prevent the presence of selection genes in the improved plants are described in Box 1. Intragenesis is an interesting NPBT concept for gene silencing. For example, in potato (Solanum tuberosum), the asparagine synthase-1 (StAst1) gene was silenced following the intragenesis concept with the aim of reducing the formation of acrylamide in potatoes during baking and frying [13]. The silencing vector comprised gene elements of different potato genes. After selection of intragenic potato lines, these were field tested and the resulting tubers showed a 70\% reduction in acrylamide levels after processing. In 2014, the US Department of Agriculture (USDA) approved the deregulation of an intragenic potato line in which, in addition to StAst1, the polyphenol oxidase-5 gene was also silenced for the prevention of black spot bruise, thus allowing the cultivation of this potato in the USA. This potato strain was also tested by the US Food and Drug Administration (FDA) for food and feed safety.

Cisgenesis may become an important approach for introducing disease resistance into elite susceptible crop cultivars, especially when the focus is on stacking multiple resistance genes. Resistance genes to late blight and scab originating from crop wild relatives have been used to produce cisgenic late blight-resistant potato [14] and scab-resistant apple [15] varieties, respectively. The performance of several cisgenic potato lines in which three different late blight resistance genes originating from Solanum stoloniferum (Rpi-sto1), Solanum bulbocastanum (Rpi-blb3) and Solanum demissum (R3a) were introduced, is currently under evaluation in field trials in the Netherlands (J. Vossen, personal communication 2014, [12]). Several cisgenic and intragenic apple lines with the apple scab resistance gene HcrVf2 originating from Malus floribunda, have been studied over a period of 3 years in a field trial in the Netherlands. Several of the cisgenic and intragenic lines showed a similar level of scab resistance to that found in a commercial apple variety in which HcrVf2 was introduced by conventional breeding [16].

As a final example, introducing an extra copy of the barley phytase gene (HVPAPhy_a) gave a 2.8-fold increase in phytase activity in the mature barley (Hordeum vulgare) grain [17]. High phytase activity is an important characteristic for grain used as feed for certain livestock because it improves phosphate uptake. This cisgenic barley line is currently being tested in field trials in Denmark and animal feed experiments are anticipated [12].

\section{Application of SSN-3}

We discuss one example of SSN-3 application that demonstrates the precision of targeted transgene integration achieved by homology-driven repair resulting in maize plants with reduced phytate content in the grains. Phytate is an indigestible, organic form of phosphorus and, therefore, an antinutritional component of feed grains. A reduced-phytate trait has agronomic and ecological significance. Application of ZFNs and DSB repair following homologous recombination using a donor DNA template (Box 2) resulted in insertional disruption of the native maize inositol-1,3,4,5,6-pentakisphosphate 2-kinase (IPK1) gene, which encodes an enzyme that catalyzes the final step in phytate biosynthesis in maize (Zea mays) seeds [10]. The donor construct, which was introduced together with sequences encoding the ZFNs, contained a promotor-less PAT gene flanked by short arms with sequences homologous to the targeted IPK1 gene. Selection of precise insertion events relied on insertion of the PAT gene behind the endogenous IPK1 promoter, resulting in expression of the marker and herbicide tolerance. Seeds of selected, herbicide-tolerant T2 plants with a biallelic targeted transgene insertion displayed reduced phytate levels with a concomitant increase in inorganic phosphate. Thus, the maize plants with a reduced phytate trait are a NPBT-product that still contains foreign sequences in the end product. 


\title{
Trends in Plant Science
}

\begin{abstract}
Box 1. Generation of Improved Plants Using NPBTs
All NPBTs make use of a genetic modification step for the introduction of recombinant DNA into the plant (Figure 1, main text). The recombinant DNA may sometimes comprise sequences that are not native to the targeted plant species and, therefore, are undesired in the final plant product. In some cases, the presence of the recombinant DNA is only required for a short period of time to induce modifications elsewhere in the DNA, as is the case when applying SSNs. In such cases, final plant products in which the recombinant DNA has not been incorporated into the plant genome may be selected directly. Other applications require the prolonged presence of the recombinant DNA, usually integrated into the plant genome. For the examples described in this review, various approaches have been used for producing plants without the presence of foreign recombinant DNA sequences in the ultimate products.
\end{abstract}

For intragenesis and cisgenesis, Agrobacterium-mediated transformation is usually used for introducing the new genes. This may involve the introduction of a selection gene for the efficient selection of transformed plants. Selection genes are often antibiotic- or herbicide-resistance genes because they are efficient for the selection of transformed plants, but they are of nonplant origin and have to be removed afterwards. In the examples of intragenesis and cisgenesis in potato, the transformation frequency was sufficiently high and no selection gene was used. Here, intragenic and cisgenic plants were selected by amplification of a unique intra- or cisgene fragment using PCR [13,14]. For the introduction of the apple scab resistance gene HcrVf2 into apple, a selection gene was used for the recovery of transformed apple shoots [16]. This gene was subsequently removed using an inducible site-specific recombinase [45]. Finally, in the example describing cisgenesis in barley, a selection gene was also used, but was introduced as a separate T-DNA, resulting in the unlinked integration of the HvPAPhy_a cisgene and the selection gene in the barley genome. After crossing, offspring were selected that inherited the desired cisgene but not the selection gene [17].

When applying SSNs, the nuclease activity encoded by these genes is only required for a limited period of time, during which the modifications are established. After this, the SSN gene is no longer needed. The final products are plants with modifications in their own DNA, but without SSN genes integrated into the genome. In the described example of maize plants with a modified IPK1 gene, mutant plants were produced using transient expression of ZFNs [10]. For this, a direct gene-transfer method using silicon carbide whiskers in embryogenic cell cultures was used and mutated maize plants without ZFN genes incorporated were directly recovered from these cell cultures. In potato, a transient expression was also applied, using protoplasts and a TALENs construct [21]. A proportion of the selected shoots that were regenerated from the protoplasts contained the intended mutations in the VInv gene, but no TALEN DNA. Such transient expression systems need specific tissue culture requirements and have so far only been developed for a limited number of species. In most of the cases described, the SSN genes are stably integrated into the plant genome, after which modified plants are recovered from the transgenic plants. After the modifications have been established, SSN-containing plants are crossed and offspring are selected that have not inherited the SSN genes. This approach was used in the examples described for targeting the Os11N3 gene in rice [18], Mlo in bread wheat [19], and the FAD2 gene in soybean [20]. Such a strategy cannot be used in crops with complex genetics, a long generation time, or that are propagated vegetatively. An alternative approach described for SSN delivery and production of mutant plants without integrated SSN genes is the use of a tobacco rattle virus (TRV)-based expression system for transient delivery of ZFNs into intact plants without the need for tissue culture [46]. The ability of TRV to move to developing buds enabled the recovery of mutated plants, without ZFN genes being integrated into the genome. The TRV virus was removed from the plant using standard virus elimination methods. Whereas the cargo capacity of TRV enables the delivery of ZFN genes as well as meganuclases [47], it is not sufficient to deliver larger genes, such as TALENs or Cas9 for the CRISPR-Cas9 system. Finally, methods for nontransgenic genome editing in plants by delivery of SSN as protein or MRNA, rather than as DNA, have been proposed, thus preventing the introduction of foreign DNA and any incorporation of SSNs into the genome [48]. We may expect more developments in this area in the future.

For ODM, synthetic oligonucleotides are introduced into plant cells, which may result in point mutations in the targeted DNA in some of the regenerated plants. The method relies on naturally occurring double-stranded breaks (DSBs) in the DNA, enabling synthetic oligonucleotides to be used as a template for repair. The synthetic oligonucleotides are not incorporated into the plant genome and are degraded in the cell. Thus, this method results in modified plants without any integrated DNA fragments.

\section{NPBT Products that Contain (Small) Modifications of Their Own DNA}

This class includes products made with one of the SSN technologies (an explanation of SSNtechnology variants is given in Box 2) or with oligo-directed mutagenesis (ODM) (Table 1, Improved plant 2). These technologies aim to induce small modifications to existing genes in the plant genome. This may result in knockout mutations (by SSN-1 through the induction of deletions leading to reading frame-shift mutations or by SSN-2 through editing an amino acid codon into a stop codon), modified gene expression patterns (by SSN-1 through the deletion of promoter elements), or changes in gene functionality (by SSN-2 through inducing single 


\title{
Trends in Plant Science
}

\begin{abstract}
Box 2. Three Variants of SSN Technology
SSNs are programmable DNA-binding proteins that bind to a predefined target DNA sequence and induce a doublestranded break (DSB). Depending on the type of native repair pathway followed for repair of the induced DNA break, three different results can be expected, designated SSN-1, SSN-2, and SSN-3 (Figure I). Following the nonhomologous endjoining repair pathway, the broken DNA ends are ligated again, which may result in inaccurate repair with (mostly) small deletions or small insertions. This is referred to as SSN-1. When SSN-1 is targeted to the protein-coding sequence of a gene, the deletions and insertions may result in frameshift mutations leading to a premature stop of translation. In the presence of a DNA repair template, the DNA break may accurately be repaired by homologous recombination (HR). Depending on the type of DNA repair template available, HR can result in small changes after repair (SSN-2) or to precise insertion of a larger DNA insert (SSN-3). SSN-2 is used to introduce small changes, such as a single base substitution in a DNA sequence. Using SSN-3, a complete new gene can be inserted at a predefined location.
\end{abstract}

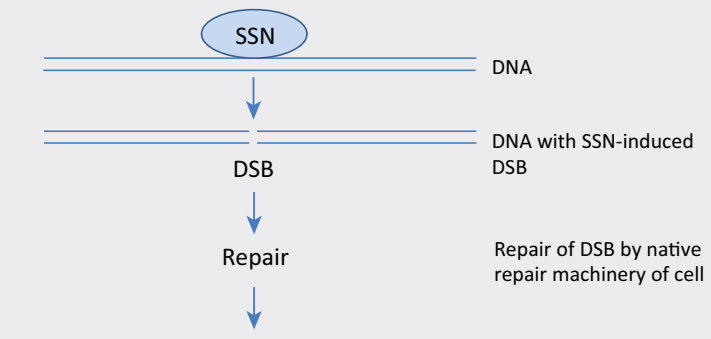

SSN-1:

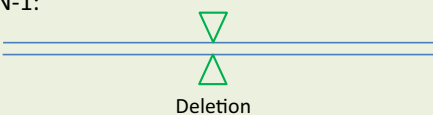

Nonaccurate repai following NHEJ

SSN-2:

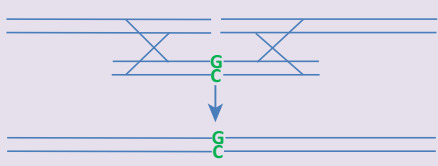

Accurate HR-directed repair using template DNA with a small change

Single base substitution

SSN-3:

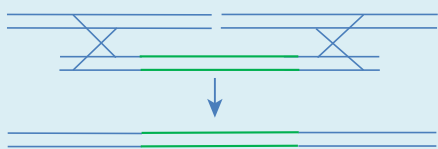

Accurate HR-directed repair using template DNA with new insert

Introduction of new insert

\section{Trends in Plant Science}

Figure I. Outline of Sequence-Specific Nuclease (SSN) Technology. SSN-1, SSN-2, and SSN-3 are SSN technology variants resulting in different types of modification. DNA is represented as double lines; modifications are shown in green. Abbreviations: DSB, double-stranded break; HR, homologous recombination; NHEJ, nonhomologous end-joining.

nucleotide substitutions or by SSN-3 through replacing gene fragments by edited ones). Once these modifications are made, the SSN genes or ODM sequences are no longer needed, and plants devoid of them have been established following various methods (Box 1).

Among the SSN technologies, SSN-1 has been rapidly adopted for switching off gene functions, and several recent examples show that it is an effective approach for the creation of new crop varieties with improved characteristics. SSN-2 and SSN-3, which rely on homology-driven repair, still have low efficiency, and we found no practical examples of NPBT products containing modifications of their own DNA based on these techniques in the recent literature. 


\section{Trends in Plant Science}

\section{Application of SSN-1 Technology}

An elegant application of SSN-1 technology was generating bacterial leaf blight resistance in rice using TALENs [18]. Bacterial leaf blight caused by Xanthomonas oryzae pathovar oryzae (commonly abbreviated as Xoo) is the most widespread pathogen in rice. For pathogenesis of several Xoo lines, the expression of the rice Os11N3 sugar transporter gene is essential. Therefore, Os11N3 is known as a susceptibility (S-) gene. To activate Os11N3, Xoo secretes effector molecules that bind to the promoter of the Os11N3 gene. Partial (five to ten base pairs) deletion of a specific region in the Os $11 \mathrm{~N} 3$ promoter using TALENs prevented Xoo from inducing Os11N3 gene expression, resulting in strong resistance to infection by some Xoo pathotypes in rice, while basic functions of the Os11N3 gene for the plant remained unaffected. The novelty of this approach was the precision, compared with inducing random mutations, by which variants of the promoter were made and tested for their effectiveness against Xoo.

In bread wheat (Triticum aestivum), SSN-1 technology was used to achieve disease resistance by means of targeted knockout of a wheat powdery mildew S-gene, called Mlo [19]. Given that bread wheat is an (allo)hexaploid species, all six Mlo alleles have to be modified to achieve the desired phenotype. Using TALENs, it was demonstrated that the three different homoeoalleles of the Mlo gene could be targeted simultaneously in the same plant, resulting in deletions in the coding sequence of Mlo. A primary transformants with the TALEN construct, containing heterozygous mutations in each of the three homoeoalleles, was self-pollinated and from the resulting progeny, TALEN-free, homozygous mutant plants with a powdery mildew-resistant phenotype were selected. This example shows the efficacy of SSN-1 technology for generation mutants in polyploid species. The same result would be almost impossible to achieve using traditional random mutagenesis approaches because the chance of achieving knockout mutations simultaneously in all homoeoalleles would be negligibly small.

Two recent examples that used SSN-1 technology to improve food quality have been described. In soybean (Glycine max), fatty acid desaturase (FAD) converts oleic acid, a monounsaturated fatty acid, into linoleic acid, which is polyunsaturated. High levels of polyunsaturated fats are undesirable in processing oils for consumption. TALENs were used to generate soybean plants in which the fatty acid profile was dramatically changed as a result of the induction of homozygous mutations in two different FAD coding genes: FAD2-1A and FAD2-1B [20]. The fact that these soybean lines were created in a single generation demonstrates the power of SSN-1 technology compared with traditional (random) mutagenesis for crop improvement. In another example using TALENs in a commercial (tetraploid) potato variety, the accumulation of reducing sugars during cold storage was switched off by knocking out all four alleles of the potato vacuolar invertase gene (VInv) [21]. Tubers from full VInv-knockout plants had undetectable levels of reducing sugars, and processed chips contained reduced levels of acrylamide. This example underlines the potential of this and other NPBTs for the direct improvement of existing elite crop varieties with complex genetics.

In a final SSN-1 example, CRISPR-Cas9 preassembled complexes of purified Cas9 protein and guide RNA were transfected into protoplast of various plant species, and plants with targeted genome modifications were regenerated from the protoplasts [22]. Following this strategy, a homolog of the Arabidopsis thaliana BRASSINOSTEROID INSENSITIVE 2 (BIN2) gene, which encodes a negative regulator of the brassinosteroid (BR) signaling pathway, was targeted in lettuce (Lactuca sativa). Of the calli that were regenerated from the protoplasts, $40 \%$ contained biallelic mutations at the target site and plants were regenerated from these. Further studies are needed to test whether the BIN2-disrupted lettuce plants have the predicted phenotype of increased BR signaling. It was concluded that, because no recombinant DNA was used in the genome-editing process, the resulting mutant plants might be exempt from current GM regulations. 


\section{Trends in Plant Science}

\section{Application of ODM}

ODM is a method based on homology-dependent repair, but the efficiency of this method is low $[23,24]$. For this reason, to date, all published examples using ODM in plants aimed at an efficiently selectable, herbicide-tolerant phenotype. Maize plants tolerant to imidazolinone herbicides have been engineered through targeted modification of the endogenous acetohydroxyacid synthase (AHAS) gene. Using a combined RNA/DNA oligonucleotide, a precise single-point mutation was copied into the maize AHAS gene at a position known to confer imidazolinone tolerance [25]. In the UK, the Advisory Committee on Releases to the Environment (ACRE), which advises the UK Government, considered that similarly produced herbicidetolerant oilseed rape plants produced by Cibus LLC have been developed using a technique that does not involve the use of recombinant nucleic acid molecules. In Germany, the relevant authority recently considered this oilseed rape to be outside the scope of German GM regulation ('Gentechnikgesetz'), under the provision that the decision could be withdrawn if the European Commission decided otherwise under Directive 2001/18/EC'.

The oilseed rape developed by Cibus is commercially available in the USA and has received Plant Novel Trait approval from the Canadian Food Inspection Agency and Health Canada. With this regulatory approval, Cibus is permitted to fully commercialize this product in Canadai".

\section{NPBT Products that Do Not Contain Altered DNA}

Some of this third class of products of NPBT facilitate breeding, such as by introducing recombinant genes that change the expression of one or more endogenous genes with the aim of speeding up breeding processes (reverse breeding or induced early flowering). Others aim at prolonged gene silencing (RNA-directed DNA methylation; RdDM), to replace alleles by more beneficial ones (SSN-3 for gene replacement) or is used to test the effect of novel genes (Agroinfiltration). In the absence of an introduced recombinant gene, or after removal (by segregation in the offspring), the final products have no change in their own DNA other than those produced by the conventional parts of the breeding process (Figure 1, Improved plant 3). Therefore, such plants are indistinguishable from conventionally bred plants. Products from grafting of a wild-type scion on a GM-rootstock are included in this class.

The NPBTs 'induced early flowering' and 'grafting on a GM rootstock' are at a stage of development where commercial application can be expected in the near future. For both NPBTs, promising examples are discussed below.

\section{Induced early flowering}

Induced early flowering has been applied to fruit trees to accelerate breeding. Fruit species, such as apple (Malus $x$ domestica) and plum (Prunus domestica), have a long generation time (5-7 years). As a consequence, fruit breeding takes a long time, especially when novel traits from related wild species are introgressed, and multiple generations are required to remove genetically linked undesired characteristics from the wild fruit species. By overexpression of the BpMADS4 gene from silver birch (Betula pendula), early flowering was successfully introduced in apple, resulting in flower induction in seedlings within the first year [26]. These early-flowering transgenic apple lines were then used for a so-called 'fast breeding' program in which disease resistance genes were stacked. In year 1, an early flowering transgenic apple line was crossed with the fire blight-resistant wild relative Malus fusca. Transgenic early flowering F1 seedlings were then backcrossed in year 2 with another apple line with apple scab and powdery mildew resistance genes and additional fire blight resistance genes. In year 3 , seedlings of this cross were selected that had inherited all three types of disease resistance gene, but not the early flowering gene. This example shows that using an early flowering-based breeding program can result in tremendous time savings when generating new fruit varieties with improved characteristics. 


\section{Trends in Plant Science}

A similar fast-breeding scheme was proposed for plum [27]. Here, the approach was called 'FasTracking' Plum Breeding. By transforming the Flowering Locus T1 (FT1) gene from Populus trichocarpa into European plum (Prunus domestica), transgenic plants were produced that flowered and produced fruits in the greenhouse within 1-10 months. Regulators at the USDA decided that plum cultivars resulting from the FasTrack breeding system fell outside the regulatory oversight of the agency, as long as the cultivars did not contain transgenes or pieces of transgenes [28].

\section{Grafting on a GM Rootstock}

There are several ways in which GM rootstocks can be useful for improving performance of nonGM scions. Using GM, the characteristics of a rootstock, such as rooting ability on heavy soils or resistance to soil-borne diseases and pests, could be improved, which would enhance the performance of a nonGM scion. Another application of GM rootstocks is to use them as a source of gene silencing through RNA interference (RNAi) [29]. Small interfering RNAs (siRNAs) are natural silencing signals in plants and artificial siRNAs can be generated in transgenic plants using RNAi-expressing vectors. The efficacy of RNAi to achieve virus resistance in wild-type sweet cherry (Prunus avium) was demonstrated in scions that were grafted onto a GM rootstock [30]. For this, a Prunus necrotic ringspot virus (PNRSV)-resistant transgenic cherry rootstock was produced by introducing an RNAi vector expressing siRNAs against the PNRSV coat protein [31]. Subsequently, a nonGM sweet cherry scion was grafted on this transgenic rootstock. The transfer of PNRSV-targeting siRNA signal molecules from the rootstock to the nontransgenic scion was confirmed and enhanced PNRSV resistance of the grafted sweet cherry scions was demonstrated. These findings showed for the first time the transfer of transgene-derived siRNAs from a GM rootstock to a nonGM scion in grafted trees and that these transferred siRNAs could enhance the virus resistance of the scions.

\section{Concluding Remarks and Perspectives}

The rapid developments in the field of NPBTs continuously add new and valuable tools to the plant breeder's toolbox. This enables the faster and more efficient creation of new crop varieties to meet the demand for sustainably improving agricultural productivity. All NPBTs have a similar aim, namely to enable crop improvements that are difficult (in terms of time and effort) to obtain through traditional breeding methods. The variety of approaches that are known as NPBTs complicates a comparison of these techniques. This is particularly an issue when considering the regulatory process $[32,33]$. To create more clarity, we provide here a classification based on the degree of genome modification in products from the different NPBTs and have ordered them in three groups. Within each group, the improved plants produced by the NPBTs have a similar degree of genetic modification, which is: (i) the addition of an extra copy of an existing gene; (ii) an induced mutation in an existing gene; and (iii) no modification at all. Using this subdivision enables us to clarify the impact that the chosen breeding technique has had on the original genome of the resulting product. For example, NPBT products from the second class are all characterized by having small mutations in their genomic DNA and these can in theory also be obtained through traditional mutagenesis. NPBT products from the third class do not contain any alterations of their genomic DNA and, therefore, are indistinguishable from traditionally bred plants.

The recent examples of NPBT applications described here mostly aim to improve crop disease resistance. NPBT are not only used to simply introduce disease-resistance genes in susceptible varieties, but also enable various advanced approaches to obtain durable resistance. For example, for durable potato cultivation, an elite potato variety can be equipped with various combinations of resistance genes by cisgenesis as described above, and the different improved variety lines may subsequently be applied in a cultivation rotation scheme to support durable disease management that can be adapted to the prevalent pathotypes of Phytophthora

\section{Outstanding Questions}

Which new developments can we expect in the field of NPBTs? The unprecedentedly quick adoption of CRISPR-Cas9 technology demonstrates the potential of current research activities in the field of NPBTs. In particular, developing methods for nontransgenic genome modification (i.e., using foreign sequences for genome modification, without having these sequences being incorporated into the genome) are of current interest.

Which new applications of NPBTs may be expected over the next few years? Most current examples of NPBT products aim at improved disease resistance and, long term, a focus on abiotic stress tolerance and quality traits may also be expected. Given that multiple pathways are often involved, it would be interesting to see whether specific combinations of targeted mutations can help improve abiotic stress tolerance.

Will products of NPBT be exempted from the current GMO legal framework? The current legal status of NPBT products is unclear and decisions regarding the legal status of products made with these techniques may differ among techniques and between legislations. We have yet to identify the best way for authorities to deal with the issue that many NPBT products are hardly or not at all distinguishable from classically bred plants. 


\section{Trends in Plant Science}

infestans. Such a breeding goal, requiring several isogenic varieties, would be impossible in potato by using traditional breeding methods. In addition, using genome-editing tools that enable the production of very precise changes, new disease resistances can be created by switching off S-genes, without affecting plant fitness [34]. Such newly discovered resistance genes can be included directly in the management system.

The examples described here demonstrate the versatile added value of NPBTs in breeding for crops with improved disease resistance. Over the longer term, a similar expansion of possibilities may also be expected for abiotic stress tolerance and important quality traits, including products with direct benefits for consumers, such as vegetables and fruits with improved nutritional quality or reduced allergenicity. Plants with reduced contents of allergens are already being produced using RNAi (e.g., apples with reduced Mal d 1 content $[35,36])$. Other targets are the major allergens in peanut [37], and the gluten genes in wheat, rye, and barley that contain celiac disease-inducing epitopes [38,39]. The acceptance by consumers increased along with increasing perceived personal benefit associated with such products, even when GM was proposed to make such hypoallergenic products $[40,41]$.

With regard to consumer acceptance, a few studies have been published about the acceptance by consumers of crops that were generated with cisgenesis. In general, these studies suggest that cisgenic procedures are seen as more acceptable than transgenic procedures [42], and it may be expected that, for other NPBTs, a comparable consumer attitude will be found. However, achieving broad social acceptance of NPBT will require a continuous open discussion with society $[4,43]$.

Some NPBT products described here have reached an advanced phase of development and commercialization can be expected in the near future. However, the current legal status of these NPBT products is unclear. Decisions regarding the legal status of products made with these techniques may differ among techniques and between legislations (also see the Outstanding Questions). The classification based on the degree of genome modification that we propose here may be helpful and it will be especially interesting to see how the authorities will deal with plants that have targeted mutations and/or (minor) deletions, or are even indistinguishable from plants derived from classical breeding. The conclusions made by the authorities are important because they will affect the time frame and the extent to which novel NPBT products will reach growers and consumers.

\section{Acknowledgement}

This work was funded by the Ministry of Economic Affairs of The Netherlands as part of the programme 'Sustainable plant production systems' (BO-20-003-006).

\section{Resources}

i www.transgen.de/pdf/zulassung/BVL_ODMCibus.pdf

ii http://cibus.com/press/press031814.php

iii www.cogem.net/index.cfm/nl/publicaties/publicatie/novel-plant-breeding-techniques

iv http://edepot.wur.nl/141713

\section{References}

1. Lusser, M. et al. (2012) Deployment of new biotechnologies in plant breeding. Nat. Biotechnol. 30, 231-239

2. Araki, M. et al. (2014) Caution required for handling genome editing technology. Trends Biotechnol. 32, 234-237

3. Jones, H.D. (2015) Regulatory uncertainty over genome editing. Nat. Plants 1, 1-3

4. Araki, M. and Ishii, T. (2015) Towards social acceptance of plant breeding by genome editing. Trends Plant Sci. 20, 145-149 


\section{Trends in Plant Science}

8. Sadelain, M. et al. (2012) Safe harbours for the integration of new DNA in the human genome. Nat. Rev. Cancer 12, 51-58

9. Cantos, C. et al. (2014) Identification of "safe harbor" loci in indica rice genome by harnessing the property of zinc-finger nucleases to induce DNA damage and repair. Front. Plant Sci. 5, 302

10. Shukla, V.K. et al. (2009) Precise genome modification in the crop species Zea mays using zinc-finger nucleases. Nature 459, 437-441

11. Ainley, W.M. et al. (2013) Trait stacking via targeted genome editing. Plant Biotechnol. J. 11, 1126-1134

12. Holme, I.B. et al. (2013) Intragenesis and cisgenesis as alternatives to transgenic crop development. Plant Biotechnol. J. 11, 395-407

13. Chawla, R. et al. (2012) Tuber-specific silencing of asparagine synthetase- 1 reduces the acrylamide-forming potential of potatoes grown in the field without affecting tuber shape and yield. Plant Biotechnol. J. 10, 913-924

14. Haverkort, A.J. et al. (2009) Applied biotechnology to combat late blight in potato caused by Phytophthora infestans. Potato Res. 52, 249-264

15. Vanblaere, T. et al. (2011) The development of a cisgenic apple plant. J. Biotechnol. 154, 304-311

16. Krens, F.A. et al. (2015) Cisgenic apple trees; development, characterization, and performance. Front. Plant Sci. 6, 286

17. Holme, I.B. et al. (2012) Cisgenic barley with improved phytase activity. Plant Biotechnol. J. 10, 237-247

18. Li, T. et al. (2012) High-efficiency TALEN-based gene editing produces disease-resistant rice. Nat. Biotechnol. 30, 390-392

19. Wang, Y.P. et al. (2014) Simultaneous editing of three homoeoalleles in hexaploid bread wheat confers heritable resistance to powdery mildew. Nat. Biotechnol. 32, 947-951

20. Haun, W. et al. (2014) Improved soybean oil quality by targeted mutagenesis of the fatty acid desaturase 2 gene family. Plant Biotechnol. J. 12, 934-940

21. Clasen, B.M. et al. (2015) Improving cold storage and processing traits in potato through targeted gene knockout. Plant Biotechnol. J. Published online April 7, 2015. http://dx.doi.org/10.1111/ pbi.12370

22. Woo, J.W. et al. (2015) DNA-free genome editing in plants with preassembled CRISPR-Cas9 ribonucleoproteins. Nat. Biotechnol. 33, 1162-1164

23. Zhu, T. et al. (1999) Targeted manipulation of maize genes in vivo using chimeric RNA/DNA oligonucleotides. Proc. Natl. Acad. Sci. U.S.A. 96, 8768-8773

24. Sauer, N.J. et al. (2015) Oligonucleotide-directed mutagenesis for precision gene editing. Plant Biotechnol. J. Published online October 27, 2015. http://dx.doi.org/10.1111/pbi.12496

25. Zhu, T. et al. (2000) Engineering herbicide-resistant maize using chimeric RNA/DNA oligonucleotides. Nat. Biotechnol. 18, 555-558

26. Flachowsky, H. et al. (2011) Application of a high-speed breeding technology to apple (Malus $x$ domestica) based on transgenic early flowering plants and marker-assisted selection. New Phytol. 192, 364-377

27. Srinivasan, C. et al. (2012) Plum (Prunus domestica) trees transformed with poplar $F T 1$ result in altered architecture, dormancy requirement, and continuous flowering. PLOS ONE 7, e40715

28. Waltz, E. (2012) Tiptoeing around transgenics. Nat. Biotechnol. $30,215-217$

29. Kalantidis, K. (2004) Grafting the way to the systemic silencing signal in plants. PLOS Biol. 2, 1059-1061
30. Zhao, D.Y. and Song, G.Q. (2014) Rootstock-to-scion transfer of transgene-derived small interfering RNAs and their effect on virus resistance in nontransgenic sweet cherry. Plant Biotechnol. J. 12 $1319-1328$

31. Song, G.Q. et al. (2013) Engineering cherry rootstocks with resistance to Prunus necrotic ring spot virus through RNAi-mediated silencing. Plant Biotechnol. J. 11, 702-708

32. Lusser, M. and Davies, H.V. (2013) Comparative regulatory approaches for groups of new plant breeding techniques. New Biotechnol. 30, 437-446

33. Podevin, N. et al. (2012) Transgenic or not? No simple answer! New biotechnology-based plant breeding techniques and the regulatory landscape. EMBO Rep. 13, 1057-1061

34. Pavan, S. et al. (2010) Loss of susceptibility as a novel breeding strategy for durable and broad-spectrum resistance. Mol. Breed. $25,1-12$

35. Gilissen, L.J. et al. (2005) Silencing the major apple allergen Mal d by using the RNA interference approach. J. Allergy Clin. Immunol. 115, 364-369

36. Dubois, A.E. et al. (2015) First successful reduction of clinical allergenicity of food by genetic modification: Mal d 1-silenced apples cause fewer allergy symptoms than the wild-type cultivar. Allergy 70, 1406-1412

37. Riascos, J.J. et al. (2010) Hypoallergenic legume crops and food allergy: factors affecting feasibility and risk. J. Agric. Food Chem. $58,20-27$

38. Gilissen, L.J.W.J. et al. (2014) Reducing the incidence of allergy and intolerance to cereals. J. Cereal Sci. 59, 337-353

39. Smulders, M.J.M. et al. (2015) Development of wheat varieties with reduced contents of celiac-immunogenic epitopes through conventional and GM strategies. In Proceedings of the 28th Meeting of the Working Group on Prolamin Analysis and Toxicity (Koehler, P., ed.), pp. 47-56, Verlag Deutsche Forschungsanstalt für Lebensmittelchemie (DFA)

40. Schenk, M.F. et al. (2011) Consumer attitudes towards hypoallergenic apples that alleviate mild apple allergy. Food Qual. Prefer. 22, 83-91

41. Schenk, M.F. et al. (2008) The influence of perceived benefits on acceptance of GM applications for allergy prevention. Health Risk Soc. 10, 263-282

42. Kronberger, N. et al. (2014) How natural is 'more natural'? the role of method, type of transfer, and familiarity for public perceptions of cisgenic and transgenic modification. Sci. Comunm. 36, 106-130

43. Palmgren, M.G. et al. (2015) Are we ready for back-to-nature crop breeding? Trends Plant Sci. 20, 155-164

44. Yamagishi, N. et al. (2014) Reduced generation time of apple seedlings to within a year by means of a plant virus vector: a new plant-breeding technique with no transmission of genetic modification to the next generation. Plant Biotechnol. J. 12, 60-68

45. Schaart, J.G. et al. (2004) Effective production of marker-free transgenic strawberry plants using inducible site-specific recombination and a bifunctional selectable marker gene. Plant Biotechnol. J. 2, 233-240

46. Marton, I. et al. (2010) Nontransgenic genome modification in plant cells. Plant Physiol. 154, 1079-1087

47. Honig, A. et al. (2015) Transient expression of virally delivered meganuclease in planta generates inherited genomic deletions. Mol. Plant 8, 1292-1294

48. Voytas, D.F. et al. Cellectis. Methods for non-transgenic genome editing in plants, WO/2014/199358 